PART V

THEORIES OF SMALL SCALE MAGNETIC FIELDS 


\title{
THEORIES OF SMALL-SCALE MAGNETIC FIELDS
}

\author{
P. A. SWEET \\ Dept. of Astronomy, The University, Glasgow, U.K.
}

\begin{abstract}
This review is concerned with the origin of the fine structure of the fields and their relationship to the heating of the solar chromosphere and corona, the structure of prominences and the production of energetic particles in solar flares. The dynamics of sunspot formation, and the large-scale structure of individual sunspots have not been dealt with, although the evolution of AR fields has been considered insofar as it affects the flare problem.
\end{abstract}

\section{The Fine-Structure at Photospheric Level}

Starting with the mean fields measured within and in the neighbourhood of spots, and in the quiet photosphere, the theoretical problem is to account for the observed fine structure and to deduce the non-radiative energy fluxes implied.

\subsection{QUIET PHOTOSPHERE}

At photospheric level there is no sharp distinction between the quiet Sun and an AR. Veeder and Zirin (1970), however, have pointed out that the fibril structure in the overlying chromospheric $\mathrm{H} \alpha$-network sets in fairly sharply at the $5 \mathrm{G}$ contour of the mean longitudinal field, and that there is no mean transverse field outside this contour. The quiet photosphere is therefore chracterized by a mean magnetic field normal to the atmosphere and of strength varying up to $5 \mathrm{G}$. The localization of this flux within relatively small areas at the junctions of three or more supergranules was first observed by Leighton et al. (1962). These concentrations, which are of the same polarity, have diameters of the order $5000-10000 \mathrm{~km}$. Within the cells a weak fine structure, of mixed polarity, of the order of $2000 \mathrm{~km}$ in diameter is present, q.v. Livingston (1968), as shown in Figure 1. There is no evidence, however, that this is correlated with the granules. The discovery by Howard (1967) of rapidly oscillating velocity fields with periods of the order of a few seconds raises the question whether yet a finer magnetic field structure may be present beyond the limit of spatial resolution.

The main intersupergranular concentrations, with peak field strengths up to $100 \mathrm{G}$, are generally attributed to convection by the horizontal motions within the supergranules as first suggested by Pikel'ner (1963) and considered quantitatively by Parker (1963). Parker's two-dimensional treatment was extended by Clark and Johnson (1967) to a three-dimensional hexagonal geometry, as shown in Figure 2. Although diffusion due to finite electrical conductivity allows a steady state to be set up in time-scales comparable with the lifetime of the supergranules, the field amplification, of the order of $R_{m}=\sigma v_{\text {hor }} L / c^{2} \sim 5 \times 10^{4}$ at photospheric level, involve field strengths far too high to be achieved by the conceivable mechanical forces available. In the above expression $L$ is the diameter of a supergranule. The concentrations must therefore be determined by the dynamics of the supergranules. A recent dynamical numer- 


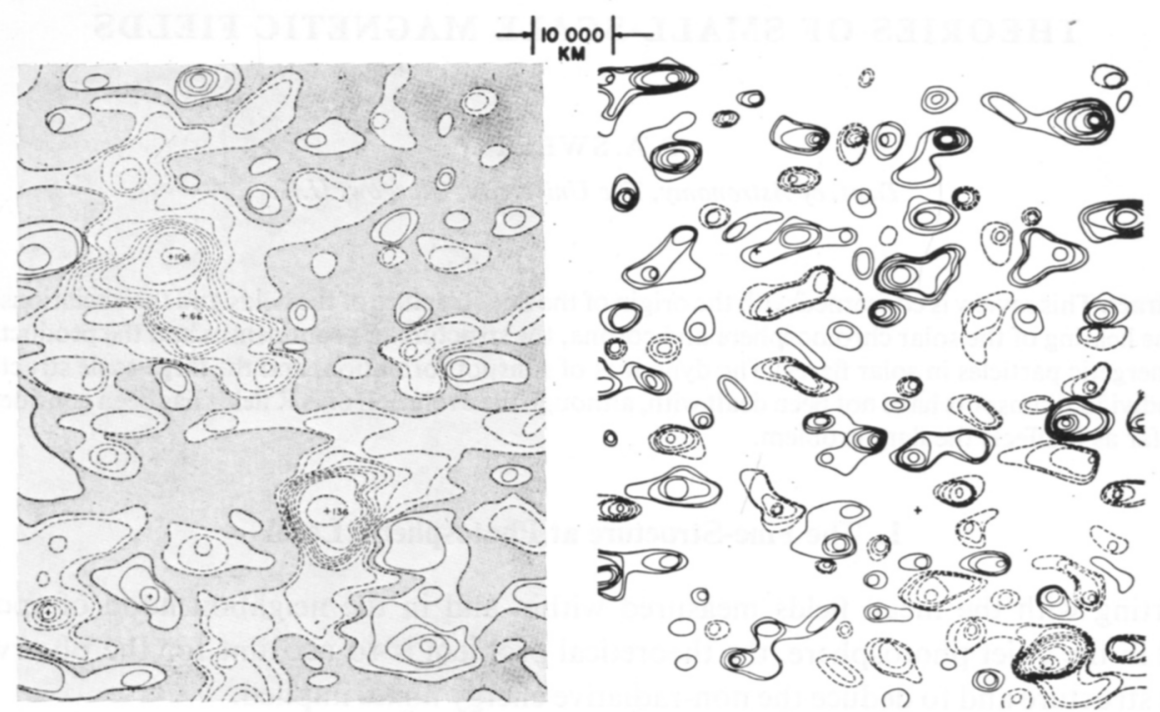

Fig. 1. Left, magnetic map of quiet photosphere. Isogauss lines are at 2, 4, 8, 16, 20, 24, 32, $64 \mathrm{G}$, with solid being $(+)$, or outgoing flux, dashed $(-)$ or ingoing flux. Right, velocity map made simultaneous to the magnetic map showing distribution of oscillatory elements. Isovelocity lines are $0.2,0.3, \ldots, 1.2 \mathrm{~km} / \mathrm{s}$ dashed is up, solid is down (Livingston, 1968).

ical model of a supergranule by Weiss (1970) has resulted in a field amplification of the order of 10 , in agreement with observation.

A considerable amount of analytical work has been done on the possible fine structure which may be produced by smaller scale turbulent motions as distinct from the large scale persistent supergranular motions. Again, dynamical effects are often neglect-

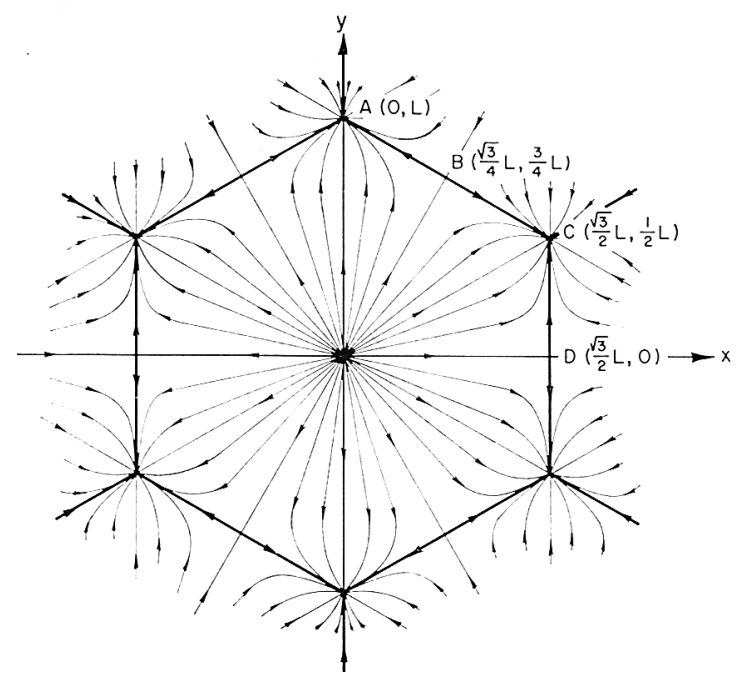

Fig. 2. Schematic of the streamlines of the horizontal motions at the top of a hexagonal supergranule (Clark and Johnson, 1967). 
ed, perhaps more justifiably in this case since in practice a statistically steady state is more likely to be determined by field diffusion than by equipartition of energy. There are, however, conflicting opinions on this point. Parker (1969) shows that if the prescribed motions are such that the velocity autocorrelation time at all wavenumbers is short compared with the time taken to travel one wavelength at the corresponding Fourier velocity amplitude then the magnetic field is amplified indefinitely at all wavenumbers. The system will therefore attain a steady state determined by the dynamics, i.e. by some form of equipartition of energy. An extension of the statistical approach has been made recently by Nagarajan (1970) to include both the dynamics and finite time ratios. The results show that, although equipartition is attained for large wavenumbers at the outset, the maximum power later shifts indefinitely towards lower wavenumbers. On the other hand a computational approach with prescribed random motions by Moss (1970) has shown that a statistically steady state is in fact reached. The rms amplification of the mean field is approximately $\frac{1}{2}\left(R_{m}\right)^{0.35}$, where $R_{m}=\tau \sigma v_{\text {turb }}^{2} / c^{2}$ and $\tau$ is the mean lifetime of a turbulent element. The amplification is almost independent of the ratio of the mean lifetime to the time taken to travel one wavelength, at least for the small values of this ratio adopted in the computation. Values $v_{\text {turb }}=0.4 \mathrm{~km} / \mathrm{s}, \sigma=10^{11}$ e.s.u. and $\tau=300 \mathrm{~s}$, typical of the photospheric granulation, lead to $R_{m} \sim 50$. The resulting amplification by granular motion would be of the order of 2 only, and not necessarily correlated with the instantaneous velocity field. A typical magnetic configuration after a statistically steady state has been reached is shown in Figure 3. This is consistent with observations. It is also to be noted that oscillations of the same amplitude, with periods of the order of $10 \mathrm{~s}$, treated as turbulence, would yield $R_{m} \sim 1$, i.e. would produce no amplification and hence no finer structure.

The reason for this apparent contradiction with the analytical approaches is not clear. It may be because Moss' model is only two-dimensional, or because the evolution has not been continued long enough in time.

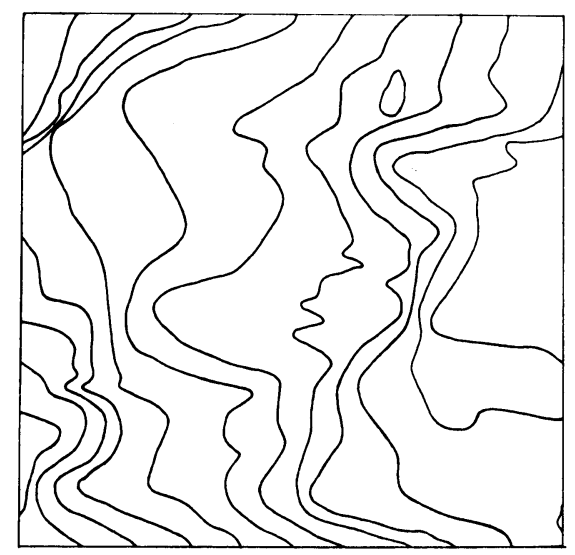

Fig. 3. Field distortion by random velocity field with $R_{m}=80$. (Ordinate is vertical direction; uniform magnetic flux is prescribed at upper and lower boundaries) (Moss, 1970). 


\subsection{ACTIVE REGION PHOTOSPHERE}

Within an AR the simultaneous longitudinal and transverse field measurements of Severny (1965) confirmed that the mean field is roughly what would be expected from flux tubes emerging through the photosphere, in the spot umbrae, into a tenuous force-free atmosphere. The field rapidly becomes transverse outside the umbral regions and areas of opposite polarity are connected. Studies of the motion of gas in the arch filaments connecting spots, by Bruzek (1969), confirm that spots grow by the emergence of subphotospheric flux tubes. The vortical structure hinted at by the chromospheric network was also confirmed, in particular by Steshenko (1969), and is consistent with the idea that the subphotospheric flux tube giving rise to the AR must have been appreciably twisted. It has been emphasized by Veeder and Zirin (1970) that the transverse field usually extends as far as the quiet photosphere. It is tempting, therefore, to think of the AR field as embedded in the general field, the magnetic surface which separates the two fields intersecting the photosphere at the boundary of the transverse field. The helmeted structures in the corona, q.v., Newkirk (1967), support this picture.

As in the quiet photosphere the mean field of an AR is shredded into flux tubes with diameters ranging from the order of $5000 \mathrm{~km}$ down to at least the limit of resolution, of the order of $1000 \mathrm{~km}$, with hints of sizes down to $300 \mathrm{~km}$. q.v., Abdusamatov and Krat (1969). The presence of transverse fields within spot umbrae themselves, as shown by Severny (1965) is striking evidence of this, as are the magnetic knots and pores with longitudinal field strengths of the order of $1000 \mathrm{G}$ outside the spots, studied by Sheeley (1967) and Steshenko (1967). These flux tubes must protrude through the tangle of flux tubes of the transverse field. The apparent motion of gas across lines of force is taken by Severny (1965) to indicate that this is occurring in the relatively field-free space between flux tubes. Regarding the connectivity of these flux tubes, the work of Beckers and Schröter (1968a) shows that part of the flux from a spot can return into the surrounding photosphere in the form of the magnetic knots. In one AR containing a unipolar spot some 3000 knots were measured, of which 2000 were of opposite polarity to the spot, 1000 were of the same polarity, and the net flux of the knots probably balanced the flux from the spot. In this case the entire flux from the spot would appear to have re-entered the photosphere as flux tubes, while the remainder of the knots returned as loops into the photosphere in the form of knots of opposite polarity. An evolutionary study of the photospheric network within an AR by Sheeley (1969) revealed a number of bright points in the photospheric emission, moving away from the spot, with velocities of the order of $1 \mathrm{~km} / \mathrm{s}$, and collecting in the surrounding supergranular network. If these are identified with flux tubes this strengthens the picture of flux tubes being convected away from the spot by the supergranular motion, and this could be the mechanism by which the spot decays. It is not obvious, however, that the disappearance of the bright points in groups indicates the mutual annihilation of flux tubes as Sheeley suggests. It could equally well be due to changes in the magnetic forces on coalescence. Regarding the areas of network which 
arise in situ as diverging bright points, it seems likely that these are produced by flux tubes emerging from below the photosphere in much the same way as the whole AR develops.

No satisfactory theory of the formation of these filaments is available. While it is true that an area of magnetic flux can be concentrated by twisting, as in the theories of Gold and Hoyle (1960) and Alfvén (1968), the result obtained by Anzer (1968), that a flux tube with a free cylindrical boundary cannot be twisted at all without destroying its cylindrical geometry, indicates that the flux tubes in previous theories are likely to be unstable. A new approach to the effect of turbulence in respect of the shredding of a magnetic field has been made by Mogilevski (1970) in terms of the statistical properties of a system of granules with isolated magnetic fields. Under solar conditions granule diameters down to $100 \mathrm{~km}$ are estimated, and the shredding could be due to collective effects of the granules. A gravitationally governed Rayleigh-Taylor instability would seem to be another possibility, but a model has not yet been worked out.

As was noted by Steshenko (1967) magnetic knots only cause a darkening of the photosphere if the field strengths exceed $1200 \mathrm{G}$. Some progress has been made towards a theoretical explanation of this by Simon and Weiss (1970) who have shown that the critical flux for penumbral formation is $10^{20} \mathrm{Mx}$, with a radius of $1500 \mathrm{~km}$, corresponding to a mean field strength of $1400 \mathrm{G}$.

There is no considerable difference in the granular motion within an AR as compared with the quiet photosphere. These motions are therefore likely to be decoupled, or almost decoupled, from the magnetic field, as in the quiet photosphere. Unlike the quiet photosphere, however, the bulk of the magnetic flux, of the order of $10^{22} \mathrm{Mx}$ is in the form of flux tubes some $1000 \mathrm{~km}$ in diameter and with field strengths of the order of $1000 \mathrm{G}$, moving outwards from the spot with transverse velocities of the order of $1 \mathrm{~km} / \mathrm{s}$. But like the large flux concentrations in the photospheric network, ohmic diffusion is unlikely to be important.

\subsection{SPOT UMBRAE}

In the umbra the mean field, of the order of $3000 \mathrm{G}$, contains an uncertain number of independent flux tubes inclined at considerable angles to the vertical, as Severny's (1965) work shows. In addition to these there appear to be gaps in the field which correspond to the umbral dots studied, notably by Beckers and Schröter (1968b). The dots are probably some $200 \mathrm{~km}$ in diameter and are as bright as the quiet photosphere. Their lifetimes are about $1500 \mathrm{~s}$. A spot umbra normally shows 20 or so dots situated largely in the outer part of the umbra. Their origin poses a difficult theoretical problem; it has been suggested by Wilson (1969) that they cannot be very much larger vertically than horizontally. If this is true they do not represent the tops of deepseated convection columns and therefore could not have the observed lifetimes without a source of energy. Joule heating would appear to be insufficient even allowing for the reduction of the effective conductivity due to the Piddington drift of the neutral gas through the plasma ions. New calculations of this conductivity by Kopecký and Kuklin (1969) and by Oster (1968) have shown values of the order of $10^{9}$ 
e.s.u. However, the dots do not appear to have large motions, and it is unlikely that they contribute to any non-radiative flux from the umbra. Spot umbrae as a whole do not, in fact, exhibit direct Doppler velocities comparable with the granular velocities of the surrounding photosphere. The turbulent velocities of the order of $2 \mathrm{~km} / \mathrm{s}$ inferred from line widths and curves of growth, q.v., Zwaan (1968) must represent a finer-scale turbulence than in the granulation.

\section{Chromospheric and Coronal Heating}

\subsection{THE QUIET ATMOSPHERE}

Observational models show that about $2 \times 10^{6} \mathrm{erg} / \mathrm{s} / \mathrm{cm}^{2}$ of non-radiative energy input is required, of which about $10 \%$ is needed for the upper chromosphere and corona. Ulmschneider (1970) shows that the lower chromosphere can be heated by non-magnetic shock-waves as Osterbrock (1961) originally suggested, although the short time interval of $10 \mathrm{~s}$ required between shocks does not agree with the granulation periods of $300 \mathrm{~s}$. The sensitivity of the heating to the interval between shocks is indicated by the curves in Figure 4. Moreover, Frazier's (1968a, b) analysis of the granulation velocities shows that the granulation flux is well below the total required. Flux with

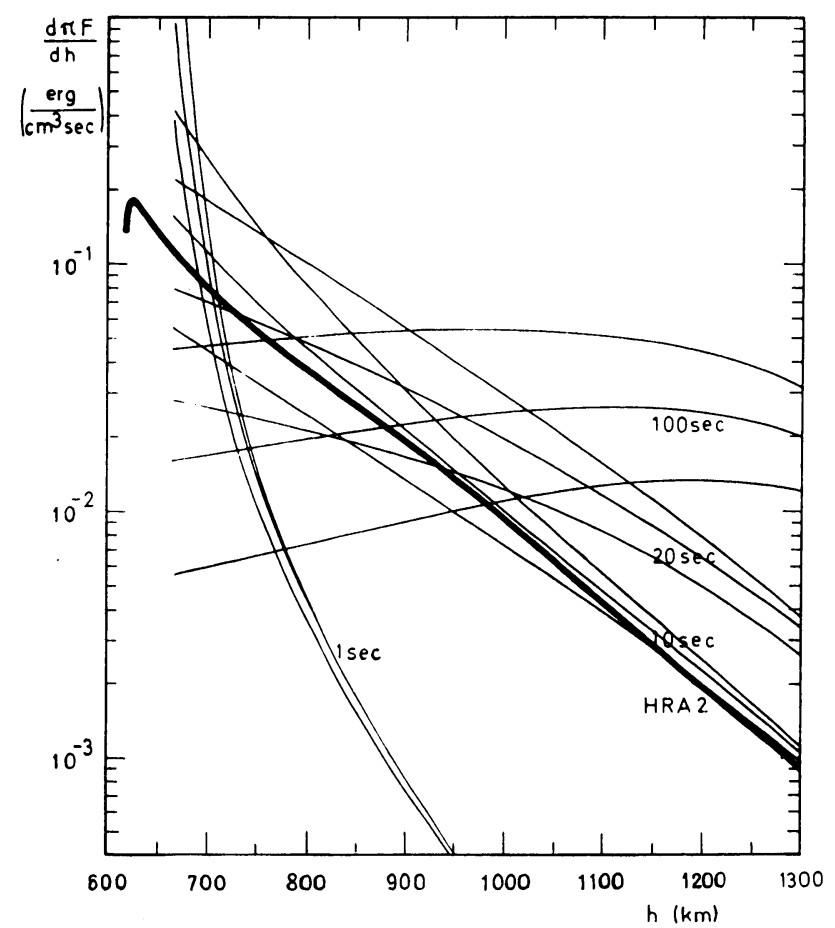

Fig. 4. Radiative energy loss (heavily drawn) compared with shock heating in the HRA2 model. There are 4 triplets of curves differing by the indicated wave periods $P$. The initial flux at $h=600 \mathrm{~km}$ (above $\tau=1$ ) in each triplet is, from bottom to top: $1.0 \times 10^{6}, 2.0 \times 10^{6}$, $4.0 \times 10^{6} \mathrm{erg} / \mathrm{s} / \mathrm{cm}^{2}$ (Ulmschneider, 1970). 
wavelengths below the present resolving power would be necessary on this theory. Certainly the $10 \mathrm{~s}$ period is closer to the theoretical period of maximum acoustic power output from the convection zone calculated by Stein (1968), and it may be that the short period oscillations observed by Howard (1967) contain the flux required.

If studied alone, and without reference to AR's, it is not entirely clear whether the quiet corona is due to magnetic effects or not. It may be significant that non-magnetic shock-wave models, notably by Kuperus (1965) and Ulmschneider (1967), cannot be extended downwards into the lower chromosphere. This may be due to a lack of understanding of the sharpness of the chromosphere - corona boundary. It was first pounted out by Kuperus and Athay (1967) that the sudden change in the temperature

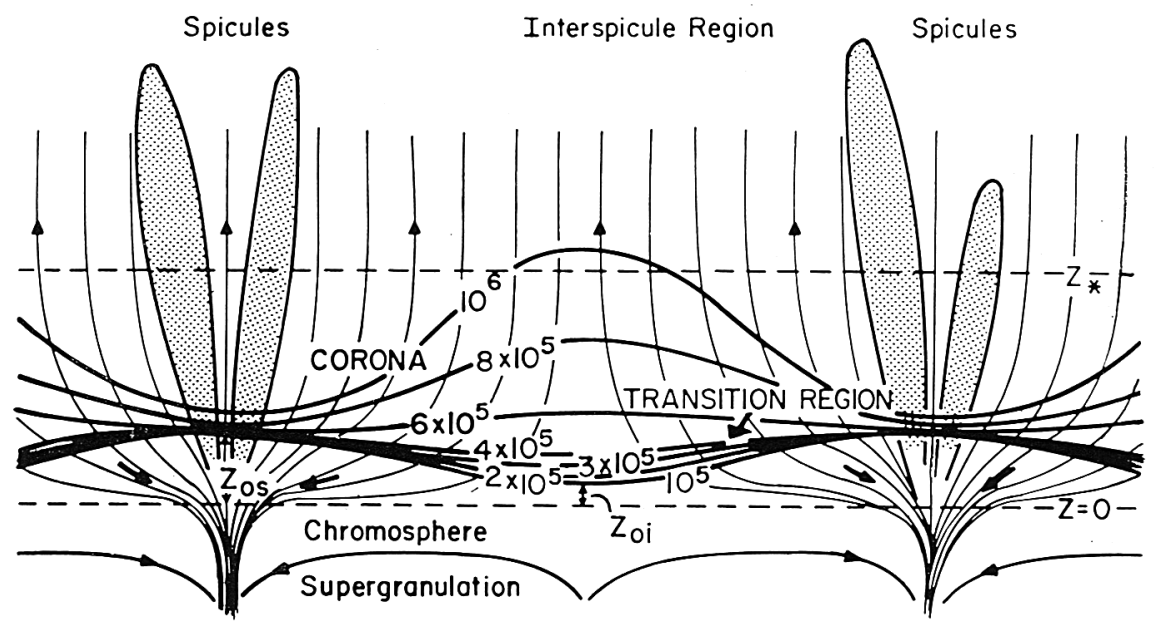

Fig. 5. A schematic cross-section of the upper chromosphere - lower corona showing magnetic field lines, isotherms and directions of the observed motions (Kopp and Kuperus, 1968).

gradient at the base of the transition region implies an input of thermal conduction energy in excess of the observed radiative loss. Any input of shock energy only aggravates this problem. Kopp and Kuperus (1968) have suggested that the channeling of the magnetic field into the intersupergranular regions concentrates the thermal conduction flux into these regions of the chromosphere, thus giving a possible origin for the spicules, as indicated in Figure 5. This mechanism depends on whether the channeling of the photospheric flux is continued up to the transition region. Clark (1968) shows that this depends sensitively on any vertical motions present, but is not inconsistent with observed supergranular motions.

A recent review of coronal heating has been given by Kuperus (1969). If coronal heating is magnetic in origin it would seem likely that the energy is propagated along the intersupergranular flux tubes. The fields in these would have the triple effect of increasing the acoustic generation rate in the convection zone, lowering the level in the chromosphere at which the magnetic stress becomes comparable with the gas 
pressure, and raising the level at which magnetohydrodynamic waves become shocks. The propagation of torsional waves along such flux tubes has been considered by Howe (1969), using an extension of the Hill spherical vortex, torsional motions being less affected by gravity. It is evident from these calculations that dissipation by the Piddington mechanism has to be allowed for before it can be certain that the wave will penetrate into the corona.

\subsection{ACtIVE REgIONS}

Although the coronal emission can increase by factors of 10-100 relative to the quiet Sun it is not known to what extent the net radiative loss from the low chromosphere is changed. Since this latter is so much the greater part of the total for the atmosphere the enhancement of the total non-radiative flux from the photosphere is uncertain. It is possible that the corona is heated independently of the low chromosphere by flux propagated along the magnetic knots. In this connection the ohmic dissipation process at the boundary of intertwined flux tubes, first suggested by Hoyle and Wickramasinghe (1961), might be worth looking into again. A sketch of the random walk of flux tubes due to supergranulation motions, which would effect such an intertwining is given in Figure 6.

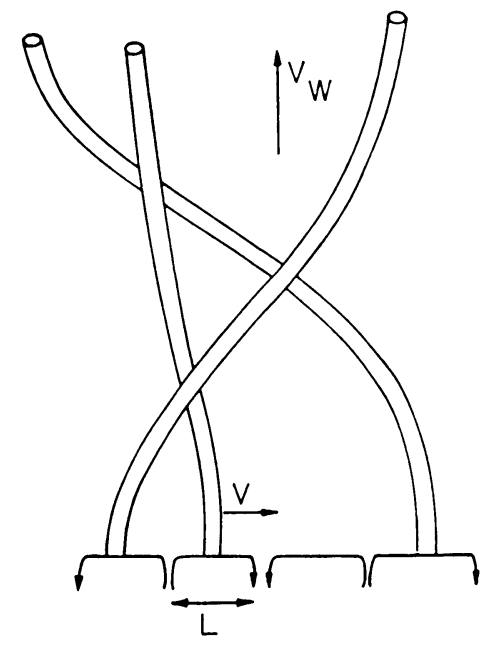

Fig. 6. Intertwining of flux tubes due to random walk produced by supergranular motions (Jokipii and Parker, 1969).

\subsection{SPOT UMBRAE}

If the turbulent velocities of $2 \mathrm{~km} / \mathrm{s}$ deduced from line widths were real and represented travelling waves, a wave flux of the order of $10^{10} \mathrm{erg} / \mathrm{s} / \mathrm{cm}^{2}$ would be implied, i.e., an appreciable fraction of the photospheric radiative flux, and more than sufficient for the entire coronal AR. It has been difficult to account for a wave flux of this order theoretically, q.v., Musman (1967). Syrovatskii and Zhugzdha (1968), however, have 
shown that if the Boussinesq approximation is dropped, a new mode of instability arises which would permit oscillatory convection. In this mode the gas oscillates in columns en tirely along the field and interacts only radiatively with adjacent columns. Thus the criterion for instability differs only slightly from the Schwarzschild criterion, e.g., for a polytropic layer with $n(=d \log \varrho / d \log T)=2$, the criterion for the second harmonic with a large field is $\gamma>1.32$ as compared with the Schwarzschild criterion $\gamma>1.5$ with no field. The usual form of the local Schwarzschild criterion with a field, q.v., Gough and Taylor (1966) is

$$
B_{v}^{2} /\left(B_{v}^{2}+\gamma P\right)>\frac{1}{\gamma}-\frac{n}{n+1},
$$

where $B_{v}$ is the vertical field strength, which of course would predict stability for a sufficiently large field. A similar study, but without an explicit application to the sunspot problem, has been made by van der Borght (1969).

\section{Prominence Fields and Stability}

\subsection{FIELD TOPOLOGY}

Studies of the field configuration in the neighbourhood of prominences, deduced partly by calculating the potential field corresponding to the observed photospheric longitudinal fluxes, and by making use of the observed longitudinal and transverse components at photospheric and chromospheric levels, have been made by Rust (1967, 1970), Iospha (1968) and Malville (1968). Figure 7 shows a typical configuration. These have confirmed the Kippenhahn and Schlüter (1957) and Dungey (1958) theoretical models of the gravitational support of a gas filament or sheet by sagging lines of force of the AR field, showing particularly that there is a component of the field along the

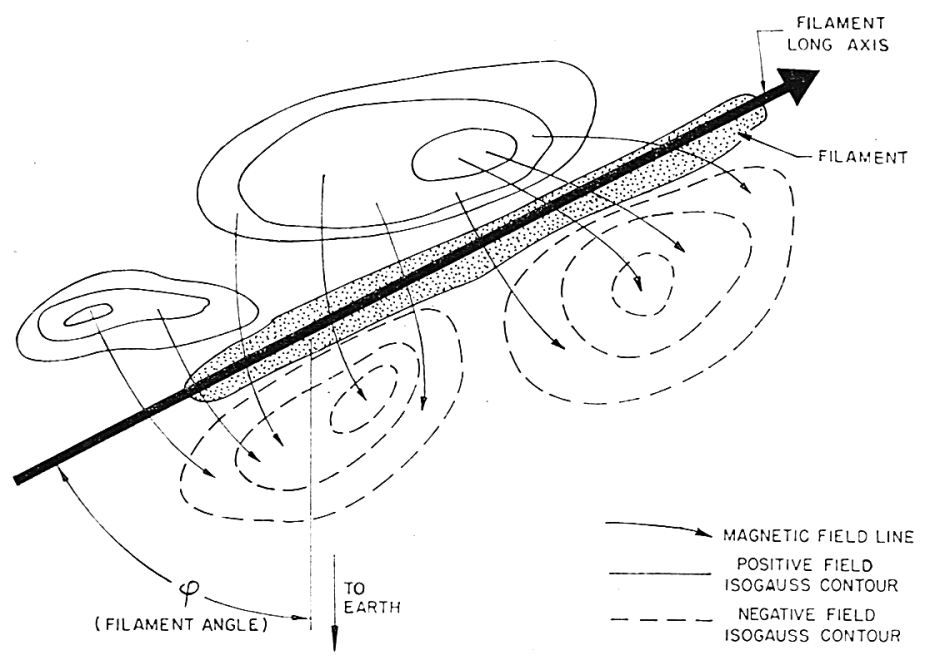

Fig. 7. Magnetic configuration in the neighbourhood of a filament inferred from photospheric magnetic fluxes (Rust, 1967). 
filament. If the field lines of the component at right angles to the filament are closed up round it the filament field is topologically a twisted flux tube embedded in the general AR field. A recent theoretical model on these lines has been by Anzer and Tandberg-Hanssen (1970). It differs from Dungey's in that, although the filament as a whole is in hydrostatic equilibrium with gravity, there is no detailed equilibrium within the filament itself.

\subsection{OVERALL STABILITY}

The important question of the dynamical stability of prominences, treated as current sheets, rather than filaments, has been dealt with rigorously by Anzer (1969). The necessary and sufficient conditions for stability are

$$
d\left|B_{x}\right| / d z \geqslant 0 \text { and } d\left(\log \left|B_{x}\right|\right) / d z \geqslant d \log \sigma / d z,
$$

where $z$ is the height coordinate, $B_{x}$ is the horizontal field crossing the sheet normally and $\sigma$ is the mass density within the sheet. It is pointed out that the first condition is often observed to be satisfied, although it is difficult to assess the second criterion, involving the density.

\subsection{INTERNAL STABILITY}

The work of Lerche and Parker (1968) in the context of the interstellar medium and the parallel work of Nakagawa (1970) applied to the prominence problem, shows that Rayleigh-Taylor instabilities put constraints on the geometry of a quiescent prominence. Figure 8 shows how an element of gas can be detached from its surroundings by a local interchange of flux tubes. This work shows that, in addition to the wellknown thermal instability of an optically thin plasma whose emission rate decreases with increasing temperature, a plane plasma supported against gravity by a magnetic field in a constant direction is always unstable to non-thermal modes. The effect of changing the direction of the field with height, the so-called onion-skin configuration, can be tested in a preliminary way by considering the instability at an interface where the field direction changes discontinuously. This situation may also arise at the interface between the prominence and its supporting vacuum AR field.

Such an interface is unstable for wavenumbers along and perpendicular, respectively,

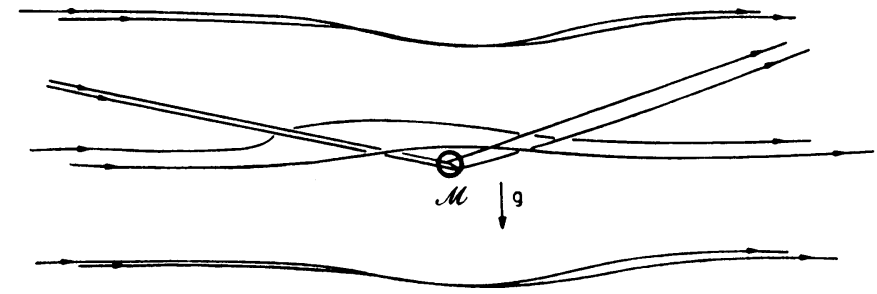

Fig. 8. Sketch of the displaced lines of force in the neighbourhood of a small element of gas $\mathscr{M}$ suspended in a magnetic field (Lerche and Parker, 1968). 
to the prominence field given by

$$
\begin{aligned}
& k_{x}<\left(V_{A}^{v} / V_{A}\right)^{2} g \cos ^{2} \theta / c^{2} \\
& k_{y}<g /\left(V_{A}^{v}\right)^{2} \sin ^{2} \theta
\end{aligned}
$$

where $V_{A}^{v}$ is the Alfvén speed corresponding to the supporting field, but with the prominence density, $V_{A}$ is the Alfvén speed within the prominence, $c$ is the thermal velocity of sound, $g$ is the acceleration of gravity and $\theta$ is the inclination of the supporting field to the prominence field. Thus, as expected, the prominence is most stable where the two fields are at right angles. The second criterion shows that instability might be suppressed if the width of the prominence were less than something of the order of $\left(V_{A}^{v}\right)^{2} / g$. This latter value would seem amply large enough to allow the observed widths of prominences.

The possibility of a microscopic plasma instability, namely the two-stream instability between electrons and ions, is raised in the current interruption theory of filament discharges by Alfvén and Carlquist (1967), who point out that in a tenuous plasma the currents induced by twisting a flux tube can involve electron-ion drifts exceeding the local velocity of sound.

\subsection{Metastability}

Finally, the usual warning should be heeded, namely, that the stability criteria as usually derived are marginal stability criteria depending on the sign of the energy taken to the second order, and are not sufficient to show whether a dynamical instability can actually arise in a given situation. For this purpose it is necessary to show that the system can be made to pass continuously from a sequence of stable configurations to a configuration in which, on continuing the sequence, no adjacent equilibrium is attainable, i.e., is in a metastable state. This requires that in marginal stability the next non-vanishing energy perturbation of higher order is negative.

\section{Flares in Relation to AR Fields}

It has long been realised that flares are associated with complex AR's during phases of rapid development, q.v. recent survey and analysis of the development of AR fields by Bappu et al. (1968) and the association with flares by Martres et al. (1968). Figure 9 shows a typical flare-producing magnetic configuration. The relationship at present is only a statistical one in the sense that within a given AR a number of flares can occur at random during a given phase, and it has been impossible to predict a flare from the instantaneous magnetic configuration. This may be partly because the only flare effect measurable with sufficient spatial resolution, i.e., the $\mathrm{H} \alpha$-emission does not occur at the seat of the energy release. Neither has it been possible to measure the field sufficiently soon before and after a flare to be able to say how much of the change observed is due to the secular evolution of the AR and how much to the flare itself. In any case it is possible for the emerging and re-entering flux to change connectivity without changing the observed longitudinal flux distribution. It is necessary 
to examine the transverse field in order to establish this. A study by Bappu et al. (1968) of the field of an AR developing on the border of a pre-existing AR shows evidence of some reconnection, as shown in the example in Figure 10.

Flares are more strongly correlated with the motions of gas in the surges and loops and with the evolution of coronal filaments, q.v., survey by Öhman (1968). Thus the sudden disappearance of filaments on the disk, and eruptive prominences at the limb,
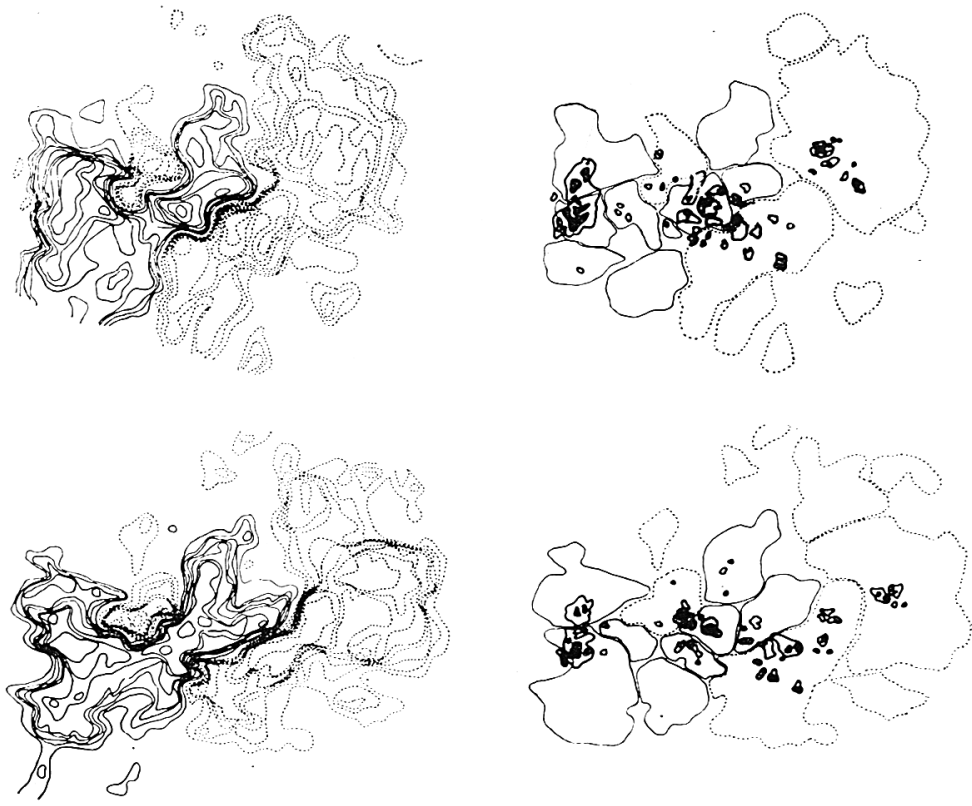

Fig. 9. An example of the development of a complex flare - producing AR, CSSAR 9. Upper, 30/09/65; lower, 1/10/65. Left, isogauss of longitudinal field; right, spots and flare positions (hatched) (Martres et al., 1968).

formerly regarded merely as the consequences of flares may now indicate that the filaments are the primary seat of the energy release and the $\mathrm{H} \alpha$-emission is due to high energy particles arriving in the photosphere or chromosphere along the field lines. The tendency of the $\mathrm{H} \alpha$-flare to be located in a series of localized regions on either side of filaments supports this view. Flare models on these lines have been put forward by Carmichael (1964), Sturrock (1968) and, in somewhat more detail in respect of the topology of the field, by Krrivský (1968) and Rust (1970). q.v., also the author Sweet (1969) for a recent review of possible mechanisms of flares. In these models it is implied that the triggering of the filament would be effected by the changes in the large-scale field of the AR, and would be governed by criteria concerning the interaction of the entire filament with the AR field, along the lines given by Anzer (1968). The current interruption theory of Alfvén and Carlquist (1967) involves the internal stability of the filament. 

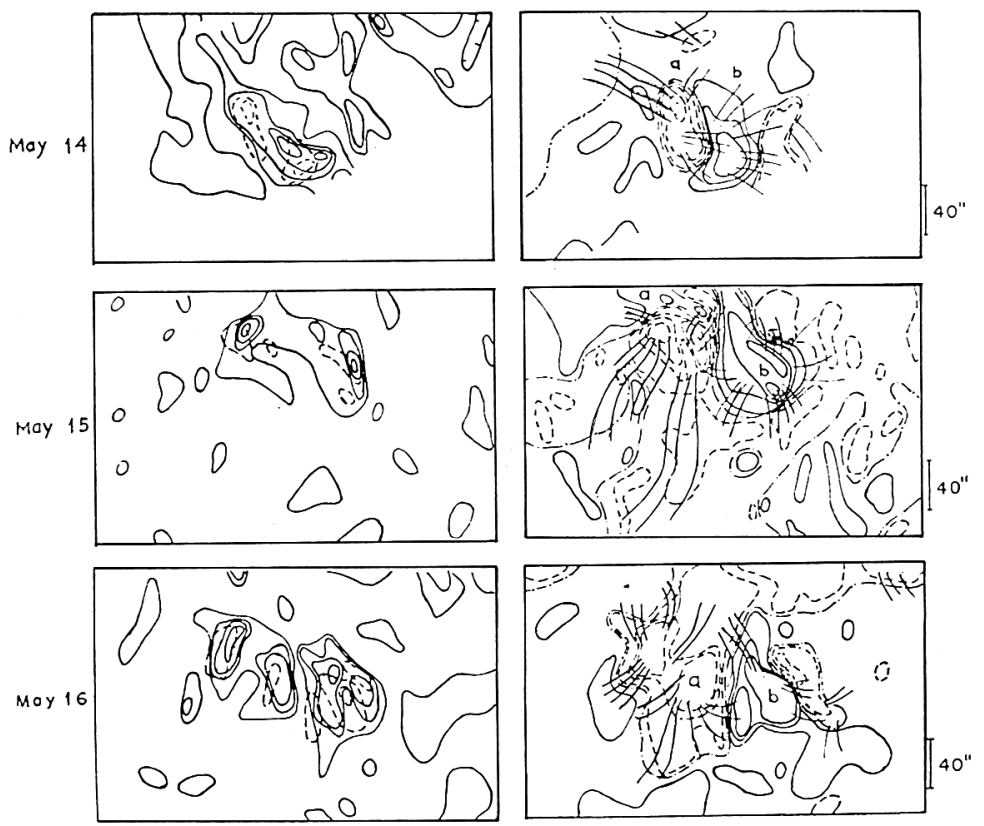

Fig. 10. Changes in the transverse field during a period of rapid evolution of an AR. Left, full lines are isogauss of the transverse field at $50 \mathrm{G}$ intervals, commencing at $100 \mathrm{G}$; dotted lines are longitudinal field concentrations. Right, isogauss of the longitudinal component and sketches of the lines of force drojected on to tangent plane. Full lines indicate $S$ polarity, dotted lines $N$ polarity (Bappu et al., 1968).

\section{Particle Acceleration}

\subsection{NUMBERS INFERRED FROM OBSERVATIONS}

The onset of a flare is characterized by radio and hard X-ray bursts indicating the production of electrons in the energy range $20 \mathrm{keV}-3 \mathrm{MeV}$. The upper end of the range is inferred, somewhat uncertainly, from the radio bursts. The lower end is uncertain since the soft end of the X-ray spectrum is of longer duration than the hard end and is of a form that could be due to thermal electrons at temperatures of the order of $10^{8} \mathrm{~K}$. The spectrum in the range $20 \mathrm{keV}-100 \mathrm{keV}$ can be represented by $N(E) \propto$ $\propto E^{-n}$ where $N(E)$ is the density of electrons with energies greater than $E$, and $n$ is in the range 2 to 4 , assuming that the hard X-rays are due to bremsstrahlung, q.v., Holt and Ramaty (1969). The spectrum falls off more rapidly at energies exceeding $100 \mathrm{keV}$. Assessments of the total number of electrons involved in the hard X-ray bursts show that it is not impossible that the total energy emitted in the flare in the form of plasma ejection, EUV and $\mathrm{H} \alpha$, subsequent to the initial burst, is derived from the energy of these high energy electrons by absorption and transformation into K.E. of mass motion, q.v., Kane and Anderson (1970). In a 2B flare the total number of high energy electrons is of the order of $10^{38}$.

In a proton flare protons up to energies of $30 \mathrm{GeV}$ are observed, the total energy 
involved being much less than that of the electrons. Owing to uncertainties of propagation in interplanetary space it is not possible to tell whether these are produced simultaneously with the electrons.

\subsection{TURBULENT ACCELERATION}

In the theories of Pneuman (1968) and Elliot (1969) high energy particles would be produced by an enhancement, within the AR, of the shockwave turbulence normally responsible for the heating of the chromosphere and corona. While there is considerable literature on the rate of acceleration of particles for given turbulence spectra, little is known about the nature of the turbulence. If, for example, the corona could be regarded as an assembly of magnetohydrodynamic shocks with a mean interval $\tau$ at any point, at a mean Mach number $M$, then statistical Fermi acceleration would apply. In this case the time taken for an acceleration from a thermal energy to a relativistic energy $E$ is given by

$$
t \simeq \frac{c \tau}{2 M V_{A}}\left(1+\frac{1}{2} \log _{e}\left(E / E_{0}\right)\right)
$$

where $E_{0}$ is the rest mass energy of the particle and $V_{A}$ is the Alfvén speed. With plausible values in the corona, $\tau=10 \mathrm{~s}, M=10$ and $V_{A}=1000 \mathrm{~km} / \mathrm{s}$, the time scale is $10^{2} \mathrm{~s}$. This is of the right order of magnitude for flares. On the other hand if the magnetohydrodynamic waves are broken down into solitons then, according to Gintsberg (1966) the maximum energy attainable by an electron is $\frac{1}{2} m_{p} M^{2} V_{A}^{2}$, where $m_{p}$ is the proton mass. Under the same conditions this latter amounts to approximately $10 \mathrm{keV}$, which is insufficient.

Again, if sufficient energy is present in the form of electron plasma waves with a uniform spectrum then, according to Pikel'ner and Tsytovich (1969), an electron with energy $E$ is accelerated according to the relation

$$
\frac{d\left(E / E_{0}\right)}{\mathrm{d} t}=\alpha\left(E / E_{0}\right)^{-1 / 2}
$$

where $\alpha \sim\left(v_{T e} / c\right)^{2} R \omega_{p e}$, and $v_{T e}$ is the electron thermal velocity, $R$ is the ratio of Langmuir to thermal energy and $\omega_{p e}$ is the electron plasma angular frequency. With values of $v_{T e}$ and $\omega_{p e}$ typical of the corona, the above mechanism is potentially capable of very rapid acceleration. The difficulty is in assessing the magnitude of the Langmuir energy.

\subsection{DiRECT ELECTRIC-FIELD ACCELERATION}

Only the component of the electric intensity in the direction of the magnetic field can be effective; the component at right angles to it merely causes the particle to participate in the mass fluid motion. The current-sheet mechanisms of Petschek (1964) and Syrovatskii (1966), and the current interruption mechanism of Alfvén and Carlquist (1967) during the flash phase, essentially provide such component fields. All three mechanisms are undoubtedly successful in producing electric currents 
intense enough to cause the electrons to drift relative to the ions with the velocity of sound. The subsequent evolution of the high current region is governed by the turbulence caused by the resulting electron-ion two-stream instability.

Since the exterior magnetic fields driving the currents are the same in both the Petschek and Syrovatskii mechanisms it would seem that they must develop in exactly the same way. The situation has been treated in some detail by Friedman and Hamberger (1969). The electric current is determined not by Coulomb collisions but by collisions with the small-scale fluctuations of electric intensity produced by the turbulence. Under these circumstances the plasma behaves as if it had the effective conductivity $0 \cdot 1\left(4 \pi n_{e} e^{2} / m_{e}\right)^{1 / 2}$ esu first established by Buneman (1959). The ambient plasma is heated on entering the high current region, the magnetic energy carried in dividing roughly equally between thermal and kinetic energy. Taking an ambient magnetic field of the order of $500 \mathrm{G}$ a temperature of $10^{8} \mathrm{~K}$, as indicated by the soft $\mathrm{X}$-rays, is therefore attainable at electron densities of the order of $4 \times 10^{11} \mathrm{~cm}^{-3}$ i.e., at chromospheric level. As in the case of ohmic currents, particles of a few times the mean thermal energy, i.e., from a threshold energy $\simeq 50 \mathrm{keV}$ are able to run away down the mean electric field. The full voltage drop available in the Petschek mechanism is of the order of $30 \mathrm{GeV}$. The authors are also able to calculate the particle spectrum.

It is tempting to believe that much the same physics might govern the Alfvén and Carlquist mechanism. There are two differences, however, which must be borne in mind. In the first place the heating of the plasma in the Petschek mechanism is derived from the magnetic energy of plasma entering the current region from outside as distinct from the former mechanism where it is heated in situ. Secondly the Alfvén and Carlquist mechanism appears to be the result of a self-triggering instability, whereas the current-sheet mechanism is not. It would therefore seem that the former

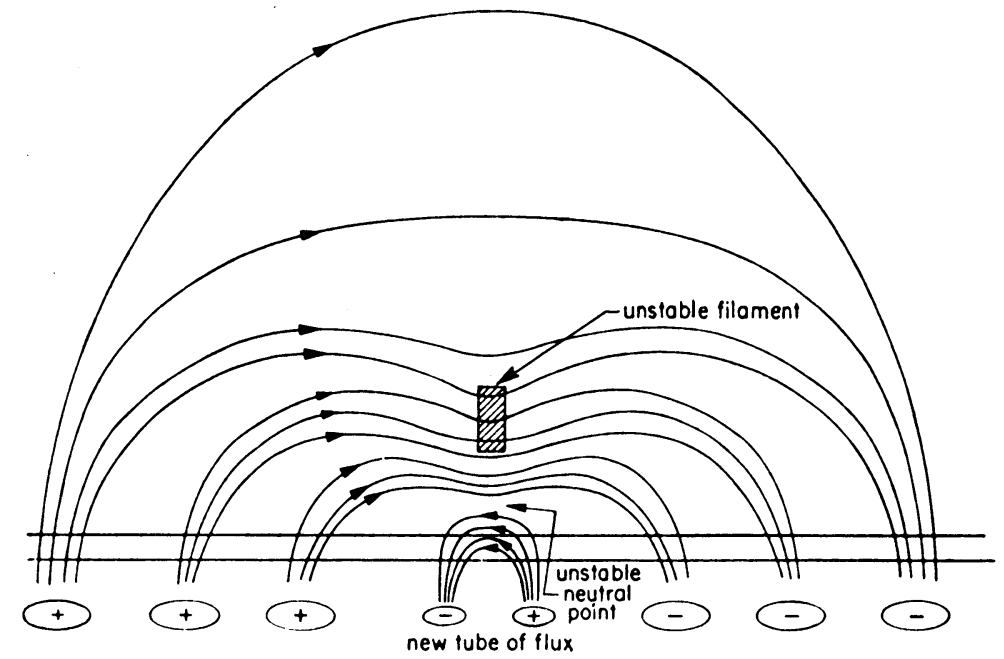

Fig. 11. Schematic representation of Kippenhahn-Schlüter-Dungey model of a prominence. The intrusion of a new flux tube from below the photosphere as shown could make the prominence unstable and also give rise to a field topology suitable for the Petschek mechanism (Rust, 1970). 
mechanism must pass the test of metastability within itself, while the latter needs an external metastability. In this connection it must be noted that the untwisting of the flux tube, which releases the energy in the former mechanism, would tend to remove the two-stream instability.

In conclusion, the Petschek mechanism, operating at chromospheric level, possibly below a chromospheric filament as in the topology of Rust (1970), shown in Figure 11, is fast enough and would appear capable of producing high energy particles in the required energy range. The total energy available in the Rust filament model, however, seems a little low for a large flare, and should be looked into more carefully. Again, the theory is incomplete in that the ability of the filament overall to become metastable has not been established.

\section{References}

Abdusamatov, H. I. and Krat, V. A.: 1969, Solar Phys. 9, 420.

Alfvén, H.: 1968, Ann. Geophys. 24, 341.

Alfvén, H. and Carlquist, P.: 1967, Solar Phys. 1, 220.

Anzer, U.: 1968, Solar Phys. 3, 298.

Anzer, U.: 1969, Solar Phys. 8, 37.

Anzer, U. and Tandberg-Hanssen, E.: 1970, Solar Phys. 11, 61.

Bappu, M. K. V., Grigorjev, V. M., and Stepanov, V. E.: 1968, Solar Phys. 4, 409.

Beckers, J. M. and Schröter, E. H.: 1968a, Solar Phys. 4, 142.

Beckers, J. M. and Schröter, E. H.: 1968b, Solar Phys. 4, 303.

Buneman, O.: 1959, Phys. Rev. 115, 503.

Bruzek, A.: 1969, Solar Phys. 8, 29.

Carmichael, H.: 1964, in AAS-NASA Symp. Phys. Solar Flares, (ed. by Hess, W. N.) U.S. Govt.

Printing Office, Washington, p. 451.

Clark, A.: 1968, Solar Phys. 4, 386.

Clark, A. and Johnson, A. C.: 1967, Solar Phys. 2, 433.

Dungey, J. W.: 1958, Cosmical Electrodynamics, p. 54, C.U.P.

Elliot, H.: 1969, in Proc. XI COSPAR Symp. Tokyo, p. 356.

Frazier, E. N.: 1968a, Astrophys. J. 152, 557.

Frazier, E. N.: 1968b, Z. Astrophys. 68, 345.

Friedman, M. and Hamberger, S. M.: 1969, Solar Phys. 8, 104.

Gintsberg, M. A.: 1966, Soviet Astron. 10, 434.

Gold, T. and Hoyle, F.: 1960, Monthly Notices Roy. Astron. Soc. $120,89$.

Gough, D. O. and Taylor, R. J.: 1966, Monthly Notices Roy. Astron. Soc. 133, 85.

Holt, S. S. and Ramaty, R.: 1969, Solar Phys. 8, 119.

Howard, R.: 1967, Solar Phys. 2, 3.

Howe, M. S.: 1969, Astrophys. J. 156, 27.

Hoyle, F. and Wickramasinghe, N. C.: 1961, Monthly Notices Roy. Astron. Soc. 123, 51.

Iospha, B. A.: 1968, in K. O. Kiepenheuer (ed.), 'Structure and Development of Solar Active Regions', IAU Symp. 35, 261.

Jokipii, J. R. and Parker, E. N.: 1969, Astrophys. J. 155, 777.

Kane, S. R. and Anderson, K. A.: 1970, Astrophys. J. 162, in press.

Kippenhahn, R. and Schluter, A.: 1957, Z. Astrophys. 43, 36.

Kopecký, M. and Kuklin, G. V.: 1969, Solar Phys. 6, 241.

Kopp, R. A. and Kuperus, M.: 1968, Solar Phys. 4, 212.

Křivský, L.: 1968, in K. O. Kiepenheuer (ed.), 'Structure and Development of Solar Active Regions', IAU Symp. 35, 465.

Kuperus, M.: 1965, Rech. Astron. Obs. Utrecht 17, 1.

Kuperus, M.: 1969, Space Sci Rev.9, 713.

Kuperus, M. and Athay, R. G.: 1967, Solar Phys. 1, 361.

Leighton, R. B., Noyes, R. W., and Simon, G.: 1962, Astrophys. J. 135, 474. 
Lerche, I. and Parker, E. N.: 1968, Astrophys. J. 154, 515.

Livingston, W. C.: 1968, Astrophys. J. 153, 929.

Malville, J. McKim: 1968, Solar Phys. 5, 236.

Martres, M., Michard, R., Soru-Iscovici, I., and Tsap, T.: 1968, Solar Phys. 5, 187.

Mogilevski, E. I.: 1971, this volume, p. 480.

Moss, D. L.: 1970, Monthly Notices Roy. Astron. Soc. 148, 173.

Musman, S.: 1967, Astrophys. J. 149, 201.

Nagarajan, S.: 1971, this volume, p. 487.

Nakagawa, Y.: 1970, Solar Phys. $12,419$.

Newkirk, G.: 1967, Ann. Rev. Astron. Astrophys. 5, 213.

Öhman, Y. (ed.): 1968, in 'Mass Motions in Solar Flares and Related Phenomena', Nobel Symp. 9, 13.

Oster, L.: 1968, Solar Phys. 3, 543.

Osterbrock, D. E.: 1961, Astrophys. J. 134, 347.

Parker, E. N.: 1963, Astrophys. J. 138, 552.

Parker, E. N.: 1969, Astrophys. J. 157, 1119.

Petschek, H. E.: 1964, in AAS-NASA Symp. Phys. Solar Flares (ed. by Hess, W. N.) U.S. Govt. Printing Office, Washington p. 426.

Pikel'ner, S. B.: 1963, Soviet Astron. 6, 757 .

Pikel'ner, S. B. and Tsytovich, V. N.: Soviet Astron. 13, 5.

Pneuman, G. W.: 1968, Solar Phys. 2, 462.

Rust, D. M.: 1967, Astrophys. J. 150, 313.

Rust, D. M.: 1970, Astrophys. J. 160, 315.

Severny, A. B.: 1965, Soviet Astron. 9, 171.

Sheeley, N. R.: 1967, Solar Phys. 1, 171.

Sheeley, N. R.: 1969, Solar Phys. 9, 347.

Simon, G. and Weiss, N. O.: 1970, Solar Phys. 13, 1.

Stein, R. F.: 1968, Astrophys. J. 154, 297.

Steshenko, N. V.: 1967, Publ. Krymsk. Astrofiz. Obs. 37, 21.

Steshenko, N. V.: 1969, Publ. Krymsk. Astrofiz. Obs. 39, 245.

Sturrock, P. A.: 1968, in K. O. Kiepenheuer (ed.), 'Structure and Development of Solar Active Regions', IAU Symp. 35, 471.

Sweet, P. A.: 1969, Ann. Rev. Astron. Astrophys. 7, 149.

Syrovatskii, S. I.: 1966, Soviet Astron. 10, 270.

Syrovatskii, S. I. and Zhugzhda, Yu. D.: 1968, Soviet Astron. 11, 945.

Ulmschneider, P. H.: 1967, Z. Astrophys. 67, 193.

Ulmschneider, P. H.: 1970, Solar Phys. 12, 403.

Van der Borght, R.: Astron. Astrophys. 2, 96.

Veeder, G. J. and Zirin, H.: 1970, Solar Phys 12, 391.

Weiss, N. O.: 1970, private communication.

Wilson, P. R.: 1969, Solar Phys. 10, 404.

Zwaan, C.: 1968, Ann. Rev. Astron. Astrophys. 6, 135.

\section{Discussion}

Wilson: Would not the oscillatory convection suggested by Syrovatskii and Zhugzdha transport too much energy into the visible region of the spot so that it would be brighter than is actually observed?

Sweet: It would not be possible to assess the flux without incorporating the mechanism into a model of the overall structure of the spot. There have certainly been difficulties in the past in achieving an energy balance in spot models.

Athay: The question of magnetic field line orientation above the supergranules is of fundamental importance to problems related to the chromosphere-corona transition region. Could you comment further on what is known, or predicted by theory, concerning the field lines over the supergranules? To my knowledge we have no direct observational evidence as to the true character of the field lines in this region.

Sweet: The height to which the flux concentrations can persist has been considered theoretically by Clark. It does seem possible from his work that the concentrations can extend to the chromosphere- 
corona transition layer. However, his work was based on a linear theory which may be difficult to apply to the large flux variations involved.

Wild: You mentioned that in both the Petschek and the Alfvén flare mechanisms there was a problem in obtaining sufficient energy release to explain the flare. Can you indicate how one estimates an upper limit to the energy available by these mechanisms?

Sweet: In both mechanisms the energy depends on the amount which can be stored by deforming the potential field into a force-free field by motions at photospheric level. It is impossible to derive this from direct observations of the field, but from a theoretical view-point it is difficult at present to see how appreciable energy can be stored without making the field unstable. It certainly seems less than the $10^{32}$ ergs required for a large flare. 